\title{
Aptidão agroclimática da cultura da videira no Estado da Bahia, Brasil
}

\author{
Antônio H. de C. Teixeira ${ }^{1}$, Renival A. de Souza ${ }^{1}$, Paulo H. B. Ribeiro², \\ Valdecira C. da S. Reis ${ }^{3} \&$ Maria das G. L. dos Santos ${ }^{4}$ \\ ${ }^{1}$ Pesquisador, Embrapa Semi-Árido, CP 23, CEP 56300-970, Petrolina, PE. E-mail: heribert@cpatsa.embrapa.br (Foto) \\ ${ }^{2}$ Embrapa Semi-Árido \\ ${ }^{3}$ Embrapa Semi-Árido \\ ${ }^{4}$ Embrapa Semi-Árido
}

Protocolo $155-8 / 11 / 2001$

\begin{abstract}
Resumo: Para delimitação das áreas com diferentes aptidões agroclimáticas para o cultivo da videira (Vitis vinifera L.) no Estado da Bahia, Brasil, foram utilizados índices baseados no balanço hídrico climático e nas temperaturas médias do ar. Inicialmente calcularam-se os balanços hídricos pelo método de "Thornthwaite \& Mather-1955", utilizando-se $120 \mathrm{~mm}$ de retenção de água no solo, com dados climatológicos das regiões de dispersão natural e de cultivo comercial da espécie. Com os dados desses balanços, determinou-se o índice hídrico anual de Thornthwaite $\left(I_{h}\right)$, caracterizando-se o grau de umidade ideal do clima para a cultura. Posteriormente, utilizando-se os valores de temperatura do ar e de precipitação pluvial mensais de 408 localidades do Estado, calcularam-se os balanços hídricos para a mesma capacidade de armazenamento de água no solo e se constatou que não há limitação térmica para o cultivo da espécie na Bahia, sendo os maiores teores de açúcar obtidos nos locais com temperaturas mais elevadas. Portanto, as zonas com aptidão plena foram subdivididas de acordo com os valores de temperatura média do mês mais quente $\left(T_{q}\right)$ de cada local considerado. A sobreposição das isolinhas de $T_{q}$ e $I_{h^{\prime}}$ no Estado resultou em quatro classes de aptidão agroclimática.
\end{abstract}

Palavras-chave: Vitis vinifera L., zoneamento agroclimático, temperatura do ar

\section{Agroclimatic aptitude of vine crop in Bahia State, Brazil}

\begin{abstract}
The use of agroclimatic indexes based on water balance and air temperature means, allowed the characterization of areas with different aptitude for grape (Vitis vinifera L.) crop growth in Bahia State, Brazil. Thornthwaite and Mather (1955) water balance for $120 \mathrm{~mm}$ soil moisture capacity, of the regions of natural dispersion and of areas of commercial crop production was used to determine the annual hydric index of Thornthwaite $\left(I_{h}\right)$ in the characterization of ideal hydric conditions of climate for the crop growth. Monthly climatic values of temperature and rainfall were used to obtain the water balance for the same soil moisture capacity for 408 locations of Bahia State. It was observed that the State has no thermal limitation for the grape growth, being higher contents of sugar obtained in the regions with higher mean summer temperatures. So, the zones of full aptitude were subdivided according to the highest monthly value of temperature $\left(T_{q}\right)$ of each place. The combination of thermal and hydric zones resulted in four classes of agroclimatic aptitude.
\end{abstract}

Key words: Vitis vinifera L., agroclimatic zoning, air temperature

\section{INTRODUÇÃO}

O gênero Vitis faz parte da família Vitaceae, que abrange mais de 90 espécies, das quais as de origem americana (Vitis labrusca L.) européia (Vitis vinifera L.) se destacam pelo valor econômico.

O cultivo da videira européia teve início na Ásia Menor, considerada região de origem da espécie. Posteriormente, foi extensivamente difundida na Europa e nas Américas (Weaver, 1976).
A uva tem dois mercados distintos: o do vinho e outros destilados alcoólicos e o da fruta de mesa e suco natural. São diferentes as características de oferta e demanda de cada um, com propriedades específicas, sem semelhança em termos de aroma, cor, paladar, tamanho, etc. O mercado brasileiro do vinho, com um consumo de nove a dez milhões de caixas por ano, chegou a ter uma participação de $35 \%$ do produto externo, em 1995. Em 1997, essa fatia diminuiu para $27 \%$. No mercado de fruta de mesa, as uvas apirenas vêm rapidamente conquistando consumidores. Na Inglaterra, algumas redes já reduzem as 
compras da uva com semente. O mercado internacional para a uva de mesa sem sementes está extremamente atraente. $\mathrm{O}$ Chile exporta, anualmente, 60 milhões de caixas de $5 \mathrm{~kg}$, com faturamento de US\$1 bilhão. Os Estados Unidos compram 40 milhões de caixas de uva chilena por ano (Uva, 1999).

No mundo, a produção de uva e de vinho ocorre em mais de 40 países e o Brasil ocupa a $17^{\mathrm{a}}$ posição no "ranking" dos produtores mundiais de vinho (Dutruc-Rosset, 1998).

Os limites de difusão do cultivo da videira nas diversas regiões do mundo, estão condicionados, particularmente, a temperatura, radiação solar, umidade atmosférica e disponibilidade hídrica no solo, que condicionam o crescimento, desenvolvimento e a produtividade (Costacurta \& Roselli, 1980).

A radiação solar atua nos processos de fotoenergia (fotossíntese) e nos processos de fotoestímulos (processos de movimento e de formação). A radiação fotossinteticamente ativa, na faixa de comprimento de onda entre 400 a $700 \mathrm{~nm}$, é fortemente absorvida e esta absorção depende do sistema de condução e do manejo cultural (Smart, 1985).

O processo de evapotranspiração da videira é determinado pela quantidade de energia disponível para o processo de vaporização da água. A radiação solar é a principal fonte dessa energia. Devido as diferenças nas posições do Sol, a intensidade de radiação incidente depende da turbidez da atmosfera e da presença de nuvens que refletem e absorvem grande parte dessa radiação (Allen et al., 1998).

A temperatura do ar interfere na atividade fotossintética das plantas, uma vez que envolve reações bioquímicas, cujos catalisadores, as enzimas, são dependentes da temperatura para expressar sua atividade máxima. As reações da fotossíntese são menos intensas em temperaturas inferiores a $20{ }^{\circ} \mathrm{C}$, intensificam-se com aumento da temperatura, atingindo o máximo entre 25 e $30^{\circ} \mathrm{C}$, voltando a cair quando a temperatura se aproxima de $45^{\circ} \mathrm{C}$. A possibilidade de cultivo da videira está estreitamente ligada à fenologia da planta. Temperaturas abaixo de $-15^{\circ} \mathrm{C}$ causam a morte das plantas e os danos causados por temperaturas elevadas são uma função de vários fatores, sendo os limites de resistência muito variáveis, situando-se entre $38 \mathrm{e}$ $50{ }^{\circ} \mathrm{C}$. A faixa de temperatura média considerada ideal para a videira situa-se entre 20 e $30^{\circ} \mathrm{C}$ (Costacurta \& Roselli, 1980).

Temperaturas abaixo de $10^{\circ} \mathrm{C}$ limitam o crescimento de brotações, induzindo a planta a entrar em período de repouso vegetativo nas regiões de clima temperado. Esse período é necessário para a formação de hormônios de frutificação, que transformam as gemas vegetativas em frutíferas (Winkler et al., 1974).

As flores começam a aparecer quando a temperatura média diária alcança $18^{\circ} \mathrm{C}$. Nos climas tropicais, o período de dormência é alcançado através do manejo de água durante o período de repouso, sendo possível obter-se produções em qualquer período do ano. Observa-se, porém, queda de rendimento nas safras iniciadas nos meses mais frios (Araújo, 1994).

Com relação à composição química da uva, não havendo excesso de precipitação pluvial, quanto mais elevada for a temperatura da região de cultivo, dentro dos limites críticos, o clima contribuirá para uma concentração maior de açúcar e menor de ácido málico nos frutos, favorecendo as produções de uva de mesa, passas e vinhos doces (Coombe, 1987; Winkler et al., 1974).
A umidade do ar durante o ciclo é muito importante, por favorecer o surgimento de doenças fúngicas. Valores elevados proporcionam o desenvolvimento de ramos vigorosos, porém a incidência de fungos é muito maior (Mota et al., 1974). Quando altos valores de umidade relativa estão associados à temperaturas elevadas, às doenças fúngicas, principalmente o míldio, provocam maiores danos econômicos, podendo inviabilizar a produção.

O déficit de pressão do vapor d'água entre a copa do parreiral e o ar vizinho, é fator determinante para a remoção do vapor. Parreirais bem irrigados em regiões áridas consomem grandes quantidades de água, devido à grande disponibilidade de energia solar e ao poder dessecante da atmosfera (Allen et al., 1998).

Em termos de exigência hídrica, a videira é muito resistente à seca, graças ao seu sistema radicular, que é capaz de atingir grandes profundidades (Costacurta \& Roselli, 1980). As regiões de cultivo incluem áreas em que a ocorrência de baixas precipitações e alta demanda evaporativa impõem o fornecimento de água através da irrigação. Tanto a deficiência como o excesso hídrico afetam, de maneira marcante, o comportamento dos estádios fenológicos da cultura da videira, comprometendo a qualidade e produtividade dos frutos. A deficiência, quando ocorre durante o período inicial de crescimento das bagas, proporciona redução no tamanho dos frutos; quando acontece durante a maturação, atrasa o amadurecimento, afetando a coloração e favorecendo a queima dos frutos, pela radiação solar. $\mathrm{Na}$ fase final de maturação o consumo hídrico da videira diminui. O excesso hídrico, combinado com temperaturas elevadas, torna a cultura muito susceptível à doenças. Para uma boa produtividade, é recomendável que o desenvolvimento vegetativo da planta ocorra em condições de escassez de precipitação pluviométrica e que as necessidades hídricas sejam satisfeitas através da irrigação, de acordo com o requerimento de água da cultura, sendo os métodos de gotejamento e microaspersão os mais utilizados (Teixeira \& Azevedo, 1996).

O Estado da Bahia apresenta grandes variações climáticas, por possuir as seguintes características: posição de transição entre tipos de clima diferentes, o Semi-Árido nordestino, o Sudeste úmido e o Centro-Oeste, com alternância de períodos secos e úmidos bem definidos; considerável extensão territorial; vasta região amplamente exposta ao oceano, sujeita aos efeitos da circulação do Atlântico; grande extensão na área inserida no polígono das secas; orientação e exposição do relevo aos sistemas de circulação atmosférica. $\mathrm{O}$ conjunto desses fatores resulta em diferentes condições de umidade do solo e do ar (Bahia, 1976).

O estudo objetivou a determinação da aptidão climática das diferentes regiões do Estado da Bahia, para o cultivo da videira irrigada, como base para um programa de expansão do seu cultivo comercial.

\section{MATERIAL E MÉTODOS}

Utilizaram-se médias dos totais mensais de precipitação pluviométrica de 408 localidades do Estado da Bahia e as temperaturas médias mensais observadas ou estimadas, correspondentes. Com relação à temperatura do ar, nos locais em que se dispunha apenas de dados de precipitação, a mesma 
foi estimada através da seguinte equação (Cavalcanti \& Silva, 1994).

$$
\begin{aligned}
\mathrm{T} & =\mathrm{A}_{0}+\mathrm{A}_{1} \lambda+\mathrm{A}_{2} \Phi+\mathrm{A}_{3} \mathrm{~h}+\mathrm{A}_{4} \lambda^{2}+\mathrm{A}_{5} \Phi^{2}+\mathrm{A}_{6} \mathrm{~h}^{2} \\
& +\mathrm{A}_{7} \lambda \Phi+\mathrm{A}_{8} \lambda \mathrm{h}+\mathrm{A}_{9} \Phi \mathrm{h}
\end{aligned}
$$

donde $\mathrm{T}$ é a temperatura média mensal, $\lambda$ a longitude, $\Phi$ a latitude, $h$ a altitude e os coeficientes $\mathrm{A}_{0}, \mathrm{~A}_{1}, \ldots, \mathrm{A}_{9}$ foram obtidos pelo método dos mínimos quadrados, totalizando 28 localidades, com dados reais de temperatura e 380 com dados estimados.

Os dados de temperatura média e dos totais pluviométricos das localidades situadas nas regiões de dispersão natural e de introdução comercial da cultura, foram obtidos de Rudloff (1981). Com esses dados, calcularam-se os balanços hídricos segundo Thornthwaite \& Mather (1955) para uma capacidade de retenção de água no solo de $120 \mathrm{~mm}$. Com as informações disponíveis na literatura e com os dados dos balanços hídricos das regiões de origem e de cultivo comercial da espécie, obtiveram-se os índices climáticos limite (Teixeira \& Azevedo, 1996) para o cultivo da espécie, os quais serviram de base para a classificação do grau de aptidão agroclimática das diversas regiões do Estado.

Os dados de área cultivada foram obtidos através do Sistema IBGE de Recuperação Automática - SIDRA (1998) e complementados com as informações do banco de dados da CODEVASF Cadastramento Frutícola (1999).

Com a utilização de um Sistema de Informações Geográficas, confeccionaram-se as cartas das isotermas dos meses mais quentes $\left(\mathrm{T}_{\mathrm{q}}\right)$ e mais frios $\left(\mathrm{T}_{\mathrm{f}}\right)$ do ano e do índice hídrico anual $\left(\mathrm{I}_{\mathrm{h}}\right)$ considerados parâmetros mais importantes na determinação do grau de aptidão agroclimática da cultura. Com base nas cartas de $\mathrm{T}_{\mathrm{q}}$ e $\mathrm{I}_{\mathrm{h}}$, definiram-se os índices-limite para as diferentes zonas de aptidão agroclimática para a cultura. Foi confeccionada ainda a carta de área cultivada com videira no Estado da Bahia.

\section{RESULTADOS E DISCUSSÃO}

Considerando-se as normais de temperatura média do ar das regiões de dispersão natural e de cultivo comercial da videira (Tabela 1) e a distribuição espacial desse parâmetro climático para os meses mais quentes $\left(\mathrm{T}_{\mathrm{q}}\right)$ e mais frios $\left(\mathrm{T}_{\mathrm{f}}\right)$ no Estado da Bahia (Figuras 1 e 2) constatou-se que, do ponto de vista térmico, não ocorrem limitações para o cultivo comercial da espécie no Estado; entretanto, não havendo excesso de precipitação pluviométrica que provoque prejuízos na produtividade nem qualidade das uvas, os locais com temperaturas mais elevadas proporcionam maiores concentrações de açúcar nos frutos.

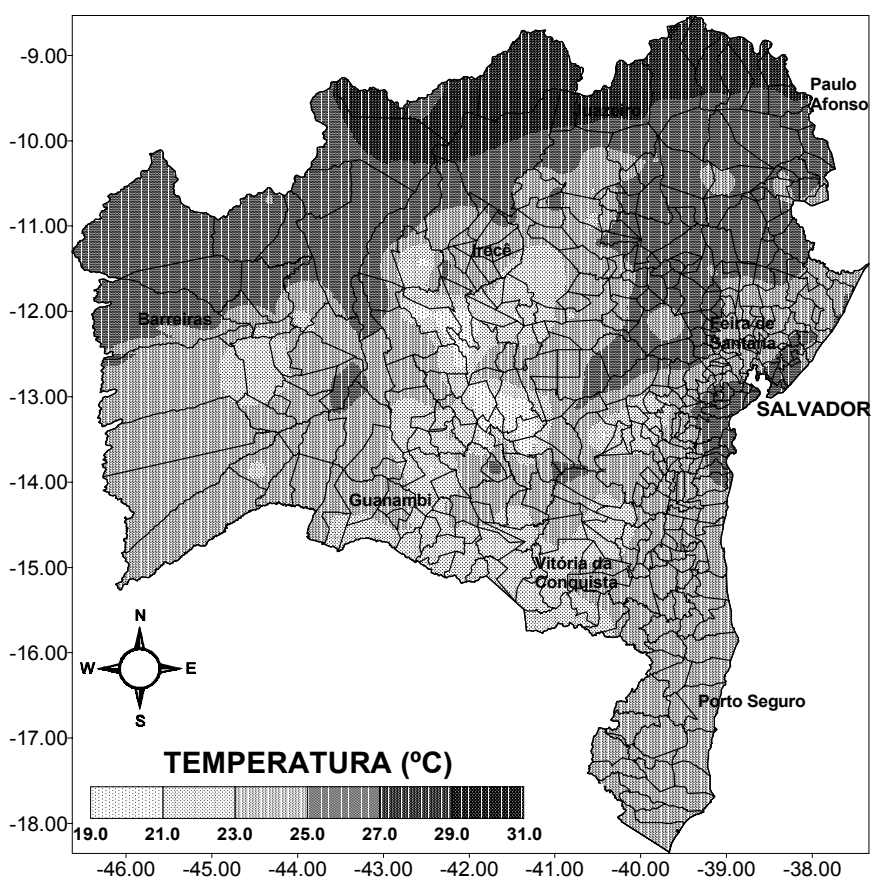

Figura 1. Temperatura dos meses mais quentes do Estado da Bahia

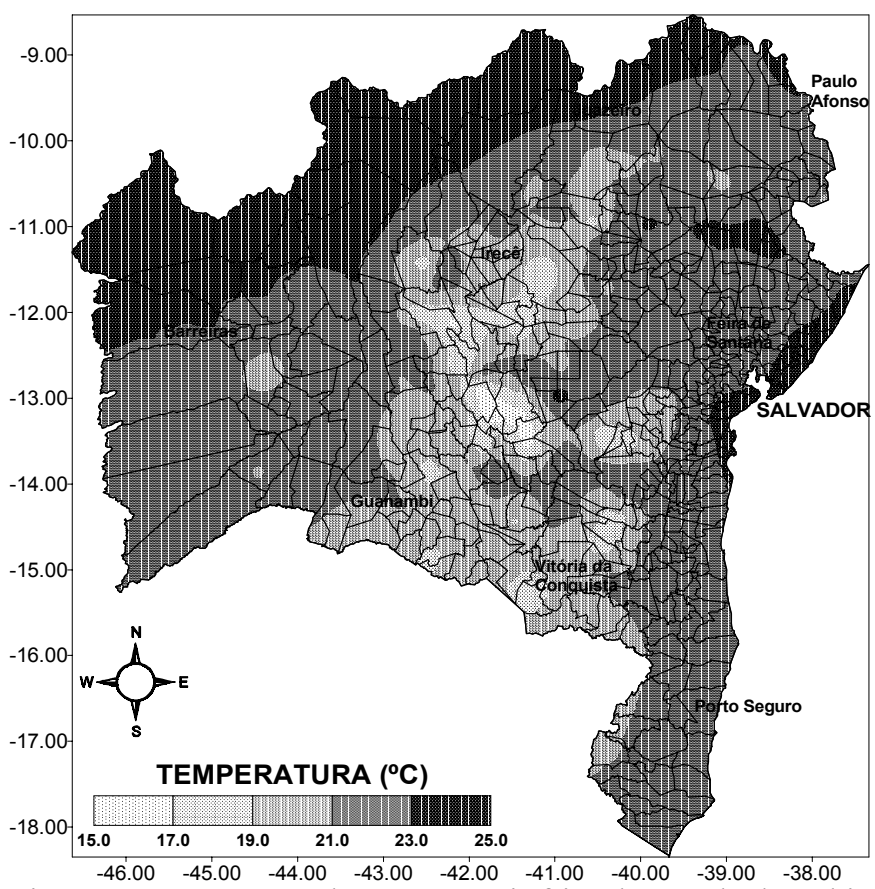

Figura 2. Temperatura dos meses mais frios do Estado da Bahia

Tabela 1. Normais anuais de temperatura média dos meses mais quentes $\left(\mathrm{T}_{\mathrm{q}}\right)$ e mais frios $\left(\mathrm{T}_{\mathrm{f}}\right)$, precipitação pluvial (P), evapotranspiração de referência $\left(\mathrm{ET}_{0}\right)$ e índice hídrico $\left(\mathrm{I}_{\mathrm{h}}\right)$ pelo método de "Thornthwaite \& Mather-1955" $(120 \mathrm{~mm})$ de localidades representativas de diversos continentes

\begin{tabular}{cccccc} 
Parâmetros & $\begin{array}{c}\text { Phenix } \\
\text { (USA) }\end{array}$ & $\begin{array}{c}\text { Mendoza } \\
\text { (Argentina) }\end{array}$ & $\begin{array}{c}\text { Ankara } \\
\text { (Turquia) }\end{array}$ & $\begin{array}{c}\text { Varna } \\
\text { (Bulgária) }\end{array}$ & $\begin{array}{c}\text { Argel } \\
\text { (Argélia) }\end{array}$ \\
\hline $\mathrm{T}_{\mathrm{q}}\left({ }^{\circ} \mathrm{C}\right)$ & 32,9 & 23,6 & 22,8 & 1,2 & 25,2 \\
$\left.\mathrm{~T}_{\mathrm{f}}{ }^{\circ} \mathrm{C}\right)$ & 10,4 & 7,6 & $-0,3$ & 22,9 & 12,2 \\
$\mathrm{P}(\mathrm{mm})$ & 184 & 197 & 344 & 474 & 691 \\
$\mathrm{ET}_{0}(\mathrm{~mm})$ & 1172 & 813 & 696 & 714 & 894 \\
$\mathrm{I}_{\mathrm{h}}$ & $-84,3$ & $-75,8$ & $-50,6$ & $-33,6$ & $-32,8$ \\
\hline
\end{tabular}


A maioria das regiões onde a videira é cultivada comercialmente, apresenta o $I_{h}$ variando entre -60 (máxima aptidão climática e um mínimo de problemas fitossanitários) e 60 (acima do qual já não é possível o cultivo comercial).

Para o Estado da Bahia, consideraram-se duas faixas de aptidão plena, com valores de $\mathrm{I}_{\mathrm{h}}$ menores que -20 , subdivididas de acordo com valores de $\mathrm{T}_{\mathrm{q}}$ menores e maiores que $28{ }^{\circ} \mathrm{C}$. Considerou-se, ainda, uma faixa de aptidão restrita $\operatorname{com~} \mathrm{I}_{\mathrm{h}}$ maior que -20 e, menor que 60 , sendo que na medida em que o grau de umidade se eleva, o número de ciclos por ano se reduz, diminuindo a produção anual. As localidades com $\mathrm{I}_{\mathrm{h}}$ superior a 60 foram classificadas inaptas sob o ponto de vista comercial.

Com a sobreposição das cartas das isotermas do mês mais quente e do índice hídrico anual, obteve-se a carta de zoneamento agroclimático para o cultivo da videira, para o Estado da Bahia (Figura 3).

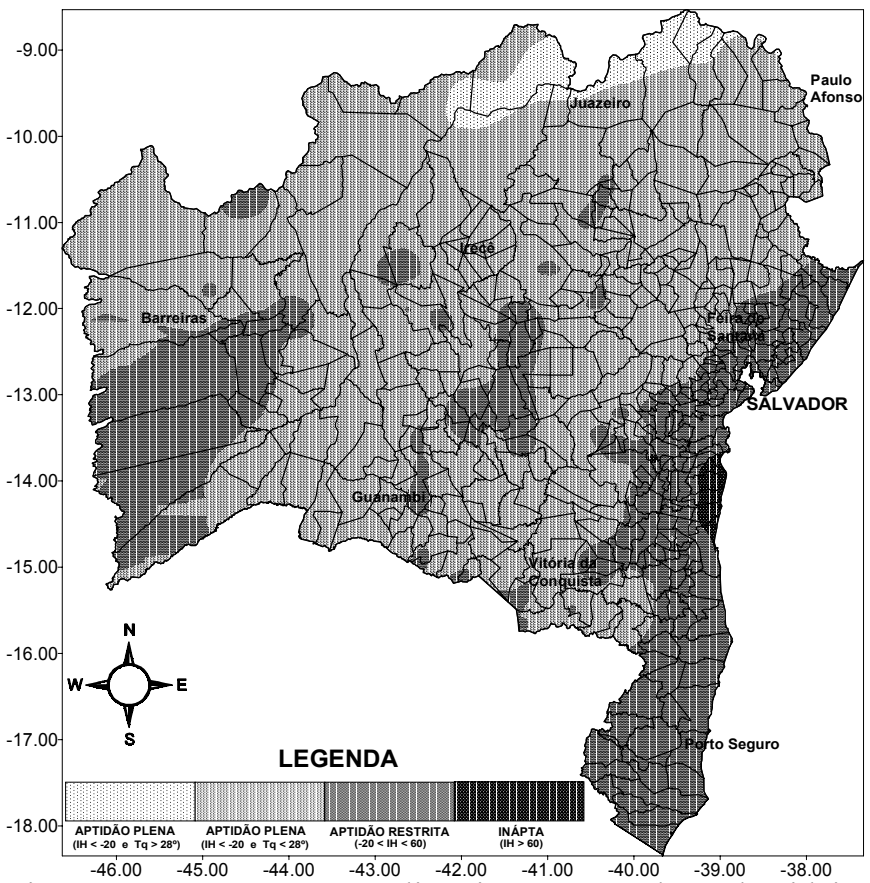

Figura 3. Zoneamento agroclimático para a cultura da videira

(Vitis vinifera L.), no Estado da Bahia

A Figura 4 apresenta o mapa temático de área cultivada com a cultura da videira. Destacam-se os municípios de Juazeiro e de Casa Nova, com áreas cultivadas na faixa de 500 a 1000 ha e de 250 a 500 ha, respectivamente, e de Sento Sé e de Curaçá, ambos na faixa de 100 a 250 ha.

Percebe-se, portanto, que em condições de irrigação, o cultivo da videira européia no Estado da Bahia tem condições de ser expandido, principalmente nos municípios de Remanso, Sento Sé, Casa Nova, Sobradinho, Juazeiro, Curaçá, Abaré, Chorrochó, Macururé e Rodelas, os quais apresentam maiores disponibilidades térmicas e baixas umidades do solo e do ar, proporcionando menor ocorrência de doenças e redução dos efeitos do excesso pluviométrico sobre o tamanho e qualidade do fruto. Esses municípios apresentam maior potencial climático para a produção de uvas de mesa e vinhos doces.

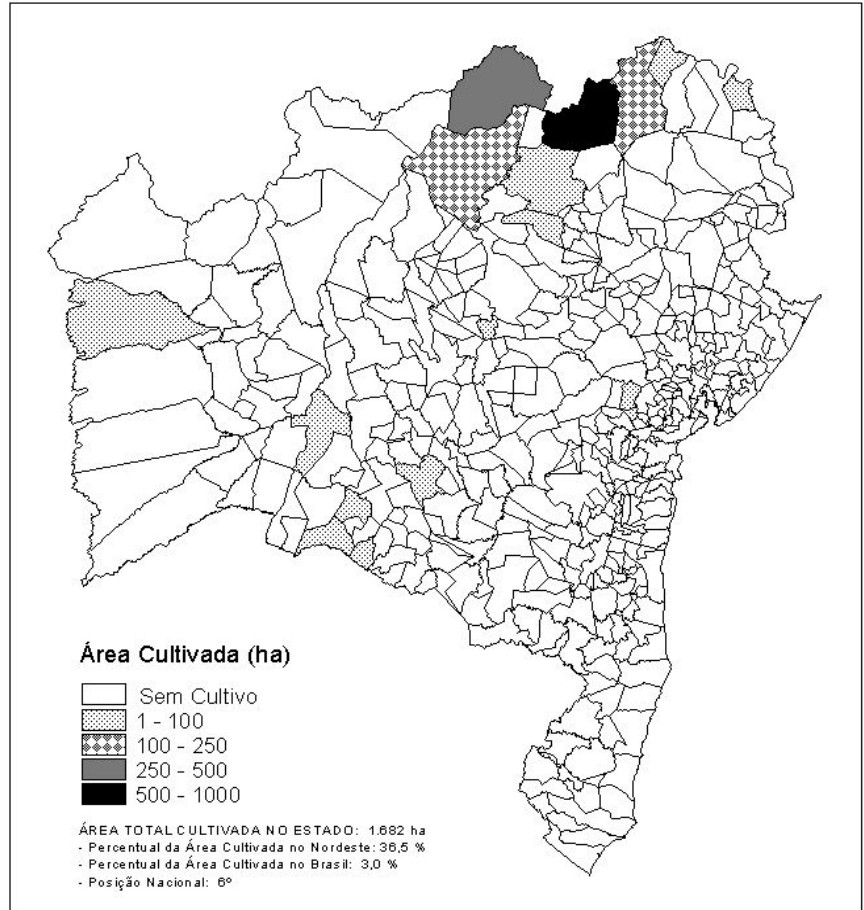

Figura 4. Zoneamento das áreas cultivadas com a cultura da videira (Vitis vinifera L.), no Estado da Bahia

\section{CONCLUSÃO}

O mapeamento das isotermas do mês mais quente e do índice hídrico de Thornthwaite, possibilitou a delimitação de zonas com diferentes aptidões agroclimáticas que, conjuntamente com outras características ecológicas, permitem um planejamento racional para o programa de expansão do cultivo da videira no Estado da Bahia.

\section{LITERATURA CITADA}

Allen, R.G.; Pereira, L.S.; Raes, D.; Smith, M. Crop evapotranspiration - guidelines for computing crop water requirements. Rome: FAO, 1998. 300p. Irrigation and Drainage, n. 56

Araujo, F.J. Table grape production in Tropical America. In: International symposium on table grape production, 1994, Anaheim, California. Proceedings. 1994.

Bahia. Secretaria do Planejamento, Ciência e Tecnologia. Atlas climatológico do Estado da Bahia: análise espacial da pluviosidade. Salvador: SEPLANTEC, 1976. 179p. Documento, 2

Cavalcanti, E.P.; Silva, E.D.V. Estimativa da temperatura do ar em função das coordenadas locais. In: Congresso Brasileiro de Meteorologia, 7, e Congresso Latino-Americano e Ibérico de Meteorologia, 1994, Belo Horizonte. Anais..., Belo Horizonte: Sociedade Brasileira de Meteorologia, 1994, p.154-157.

CODEFASF/Sede/DPOI - Companhia de Desenvolvimento do Vale do São Francisco. Cadastramento Frutícola. Versão 1.06. Brasília, 1999.

Coombe, B.G. Influence of temperature on composition and quality of grapes. Acta Horticulturae, Wageningen, n.206, p.23-35, 1987. 
Costacurta A.; Roselli G. Critères climatiques et edaphiques pour l'établissement des vignobles. Bulletin de l' O. I. V., Paris, v.53, n.596, p.783-786, 1980.

Dutruc - Rosset, G. Situation et statistiques du secteur vitivinicole mondial en 1996. Bulletin de l'O.I.V., Paris, n.803-804, p.99-175, 1998.

IBGE - Instituto Brasileiro de Geografia e Estatística. Sistema IBGE de Recuperação Automática. Rio de Janeiro: SIDRA, 1998.

Mota, F.S. da; Bersdorf, M. I. C.; Acosta, M. J. C.; Motta, W.A.; Westphalen, S.L. Zoneamento climático do Rio Grande do Sul para a videira européia. In: Conferência Latinoamericana do vinho e da uva, 6, 1974, Caxias do Sul. Ata das reuniões... Caxias do Sul: OLAVU. 1974.

Rudloff, W. World-climates with tables of climatic data and pratical suggestions. Stuttgart: Wissenschaftiliche, 1981.632p.
Smart, R.E. Principle of grapevine canopy management microclimate with implications for yield and quality. A review. American Journal of Enology and Viticulture, Lockford, v.336, n.3, p.230-239, 1985.

Teixeira, A.H. de; Azevedo, P.V. de. Zoneamento agroclimático para a videira européia no Estado de Pernambuco, Brasil. Revista Brasileira de Agrometeorologia, Santa Maria, v.4, n.1, p.137-141, 1996.

Thornthwaite, C.W., Mather, J.R. The water balance., Laboratory of Climatology, Centerton, v.8, n.1, p.1-14, 1955.

Uva: Muda a preferência do consumidor europeu. Agrianual, São Paulo, p.507-520, 1999.

Weaver, R. J. Grape growing. New York: J. Wiley, 1976, 371p.

Winkler, A.J.; Cook, J.A.; Kliwer, W.M. General viticulture. 2. ed. Berkeley: University of California Press, 1974. 710p. 